\title{
Real numbers with polynomial continued fraction expansions
}

\author{
by
}

\section{J. MC Laughlin and Nancy J. Wyshinski (Hartford, CT)}

1. Introduction. A polynomial continued fraction is a continued fraction $\mathrm{K}_{n=1}^{\infty} a_{n} / b_{n}$ whose partial numerators and denominators are integers and where, for some tail of the continued fraction, $a_{n}=f(n), b_{n}=g(n)$ for some polynomials $f(x), g(x) \in \mathbb{Z}[x]$.

One reason polynomial continued fractions are of interest is that sometimes a famous number whose regular continued fraction expansion does not have a predictable pattern can be expressed as polynomial continued fractions (so that the partial quotients do have a predictable pattern). For example the first continued fraction giving values for $\pi$ (due to Lord Brouncker, first published in [11]) is of this type:

$$
\frac{4}{\pi}=1+\stackrel{\infty}{K}_{n=1}^{\infty} \frac{(2 n-1)^{2}}{2} .
$$

Another reason polynomial continued fractions are interesting is that the elements are integers and standard theorems can sometimes be used to decide if a number is irrational. For example, there is the following criterion given by Tietze, extending the famous Theorem of Legendre (see Perron [9, pp. 252-253]):

TIETZE'S CRITERION. Let $\left\{a_{n}\right\}_{n=1}^{\infty}$ be a sequence of integers and $\left\{b_{n}\right\}_{n=1}^{\infty}$ be a sequence of positive integers, with $a_{n} \neq 0$ for any $n$. If there exists a positive integer $N_{0}$ such that

$$
\left\{\begin{array}{l}
b_{n} \geq\left|a_{n}\right|, \\
b_{n} \geq\left|a_{n}\right|+1 \quad \text { for } a_{n+1}<0
\end{array}\right.
$$

for all $n \geq N_{0}$ then $\mathrm{K}_{n=1}^{\infty} a_{n} / b_{n}$ converges and its limit is irrational.

2000 Mathematics Subject Classification: Primary 11A55.

Key words and phrases: continued fractions. 
Here we use the standard notations

$$
{\underset{\mathrm{K}}{\mathrm{K}} \mathrm{N}}_{\frac{a_{n}}{b_{n}}}:=\frac{a_{1}}{b_{1}+\frac{a_{2}}{b_{2}+\frac{a_{3}}{b_{3}+\cdots+\frac{a_{N}}{b_{N}}}}}=\frac{a_{1}}{b_{1}+\frac{a_{2}}{b_{2}+}} \frac{a_{3}}{b_{3}+} \cdots \frac{a_{N}}{b_{N}} .
$$

We write $A_{N} / B_{N}$ for the above finite continued fraction written as a rational function of the variables $a_{1}, \ldots, a_{N}, b_{1}, \ldots, b_{N}$. Then $A_{N}$ is the $N$ th canonical numerator and $B_{N}$ is the $N$ th canonical denominator.

It is elementary that the $A_{N}$ and $B_{N}$ satisfy the following recurrence relations:

$$
A_{N}=b_{N} A_{N-1}+a_{N} A_{N-2}, \quad B_{N}=b_{N} B_{N-1}+a_{N} B_{N-2} .
$$

By $\mathrm{K}_{n=1}^{\infty} a_{n} / b_{n}$ we mean the limit of the sequence $\left\{A_{n} / B_{n}\right\}$ as $n$ goes to infinity, if the limit exists.

This type of continued fraction seems to have been studied systematically for the first time in Perron [9], where degrees through two for $a_{n}$ and degree one for $b_{n}$ are studied. Lorentzen and Waadeland [7] also study these cases in detail and they evaluate all such continued fractions in terms of hypergeometric series. There is presently no such systematic treatment for cases of higher degree.

Ramanujan also gave several continued fraction identities in which the free parameters can be specialized to integers to give the values of polynomial continued fractions. An example of such a continued fraction is the following entry from Chapter 12 in the second notebook (see [2, p. 119]):

ENTRY 13. Let $a, b$ and $d$ be complex numbers such that either $d \neq 0$, $b \neq-k d$, where $k$ is a non-negative integer, and $\operatorname{Re}((a-b) / d)<0\left(^{1}\right)$, or $d \neq 0$ and $a=b$, or $d=0$ and $|a|<|b|$. Then

$$
a=\frac{a b}{a+b+d}-\frac{(a+d)(b+d)}{a+b+3 d}-\frac{(a+2 d)(b+2 d)}{a+b+5 d}-\cdots
$$

In [4], the first author and Douglas Bowman examined certain classes of polynomial continued fractions in which the $a_{n}$ and $b_{n}$ were of arbitrarily high degree. One phenomenon we observed was the existence of infinite families of in-equivalent polynomial continued fractions in which each continued fraction converged to the same limit. To illustrate this phenomenon, we cite the following example from [4]:

EXAMPLE 1. Let $f_{n}$ be a polynomial in $n$ such that $f_{n} \geq 1$ for $n \geq 1$ and let $m$ be a positive integer. Then

$\left({ }^{1}\right)$ This condition is incorrectly given as " $\operatorname{Re}((a-b) / d)>0$ " in [2]. 


$$
\stackrel{\infty}{K}_{n=1}^{\infty} \frac{f_{n}\left(\left(n^{2}+3 n+2\right) n m+1\right)+2 m n^{2}+6 m n+4 m-1}{f_{n}\left(\left(n^{2}-1\right) n m+1\right)+2\left(n^{2}-1\right) m-2}=6 m+1 .
$$

One reason to study infinite families of polynomial continued fractions in which each continued fraction converges to the same limit is that hopefully one might arrive at a classification of a "base set" such that every convergent polynomial continued fraction has the same limit as one of the continued fractions in the base set. One might further hope that every convergent polynomial continued fraction could be shown to have the same limit as some polynomial continued fraction which is at most quadratic in the numerator and at most linear in the denominator, since the limits of these types of continued fraction can be determined as ratios of hypergeometric series. It would be interesting to either see a proof of this or produce a counterexample.

In [4], our results were derived from a theorem of Pincherle (Theorem 1 below) and a variant of the Euler transformation (see equation (3.2)). In the present paper, we study this phenomenon in greater depth, considering not only further applications of Pincherle's theorem and the Euler formula, but also an extension of Euler's formula, an extension of the corresponding transformation that equates infinite products and continued fractions, extensions and contractions of continued fractions and the Bauer-Muir transformation.

As special cases of our results, we give the following examples (proofs are found throughout the paper).

EXAMPLE 4 . Let $c_{n}$ be any polynomial in $n$ such that $c_{n} \geq 2$ for $n \geq-1$. Then

$$
1=\frac{c_{0}+c_{1}^{2}}{c_{1}^{2}}+\frac{c_{0}\left(c_{1}+c_{2}^{2}\right)}{c_{2}^{2}}+\cdots+\frac{c_{n-2}\left(c_{n-1}+c_{n}^{2}\right)}{c_{n}^{2}}+\cdots
$$

ExAmPle 5. Let $A$ be a positive integer. Then

$$
\begin{aligned}
\frac{\pi}{4}= & \frac{1}{A}+\frac{1}{1}-\frac{4+A}{4-2 A} \\
& +\underset{n=3}{\stackrel{\infty}{K}} \frac{(2 n-3)(2 n-5)[A(2 n-7)+4(n-3)][A(2 n-3)+4(n-1)]}{2 A(2 n-5)+4(2 n-3)} .
\end{aligned}
$$

ExAMPLE 6 . If $\zeta(n)$ is the Riemann zeta function and $A$ is a positive integer, then

$$
\begin{aligned}
\zeta(11)= & \frac{1}{A}+\frac{2 A-1}{2 A}-\frac{2 A\left(3 A-2^{10}\right)}{3 A\left(1+2^{11}\right)-2^{12}} \\
& +{ }_{n=3}^{\infty} \frac{-n(n-1)^{21}\left[A(n-1)-(n-2)^{10}\right]\left[A(n+1)-n^{10}\right]}{A(n+1)\left[(n-1)^{11}+n^{11}\right]-2 n^{11}(n-1)^{10}} .
\end{aligned}
$$


ExAmple 7. If $A$ is any integer different from -7 , then

$$
\begin{aligned}
\sqrt[5]{\frac{12}{7}=} & \frac{A+7}{7}+\frac{7(11 A-7)}{-4 A+56} \\
& +{ }_{n=3}^{\infty} \frac{7(5 n-11)(n-2)[(12 n-37) A-7][(12 n-13) A-7]}{-2 A\left(12 n^{2}-31 n+16\right)+14(2+n)} .
\end{aligned}
$$

EXAMPLE 8. For each integer $A$,

$$
\begin{aligned}
& \frac{3 \sqrt{3}}{2 \pi}=1+A+\frac{-10 A-2}{18}+\frac{2736 A+432}{-692 A-132} \\
& +\mathrm{K}_{n=3}^{\infty} \frac{9 n(1-n)(3 n-2)(3 n-4)[(1+9 A) n+A+1][(1+9 A) n-17 A-1]}{-8(1+A)+2(5+9 A) n+144 A n^{2}-18(1+9 A) n^{3}} .
\end{aligned}
$$

EXAmple 9 . If $A$ is a non-negative integer, then

$$
\begin{aligned}
e=2 & +\frac{1}{1+A}+\frac{1-2 A(1+A)}{2(1+A)}+\frac{2(1-3 A(1+A))}{3-5 A-6 A^{2}} \\
& +{ }_{n=4}^{\infty} \frac{(n-1)[1-n A(1+A)][1-(n-2) A(1+A)]}{n-(n(n-1)-1) A-n(n-1) A^{2}} .
\end{aligned}
$$

We begin with some applications of Pincherle's theorem.

\section{Pincherle's theorem}

Theorem 1 (Pincherle [10]). Let $\left\{a_{n}\right\}_{n=1}^{\infty},\left\{b_{n}\right\}_{n=1}^{\infty}$ and $\left\{G_{n}\right\}_{n=-1}^{\infty}$ be sequences of real or complex numbers satisfying $a_{n} \neq 0$ for $n \geq 1$ and for all $n \geq 1$,

$$
G_{n}=a_{n} G_{n-2}+b_{n} G_{n-1} .
$$

Let $\left\{B_{n}\right\}_{n=1}^{\infty}$ denote the denominator convergents of the continued fraction $\mathrm{K}_{n=1}^{\infty} a_{n} / b_{n}$. If $\lim _{n \rightarrow \infty} G_{n} / B_{n}=0$ then $\mathrm{K}_{n=1}^{\infty} a_{n} / b_{n}$ converges and its limit is $-G_{0} / G_{-1}$.

In [4] we looked for solutions where $a_{n}$ and $b_{n}$ were polynomials. We then showed that the corresponding $B_{n}$ and $G_{n}$ in the statement of the theorem satisfied $\lim _{n \rightarrow \infty} G_{n} / B_{n}=0$ by using some easily deduced facts about the growth of $B_{n}$ :

(i) Let $a_{n}$ and $b_{n}$ be non-constant polynomials in $n$ such that $a_{n} \geq 1$, $b_{n} \geq 1$ for $n \geq 1$, and suppose $b_{n}$ is a polynomial of degree $k$. If the leading coefficient of $b_{n}$ is $D$, then given $\varepsilon>0$, there exists a positive constant $C_{1}=C_{1}(\varepsilon)$ such that $B_{n} \geq C_{1}(|D| /(1+\varepsilon))^{n}(n !)^{k}$.

(ii) If $a_{n}$ and $b_{n}$ are positive numbers $\geq 1$, then there exists a positive constant $C_{3}$ such that $B_{n} \geq C_{3} \phi^{n}$ for $n \geq 1$, where $\phi$ is the golden ratio $(1+\sqrt{5}) / 2$. 
The drawbacks to this approach are, firstly, the difficulty in finding solutions to equation (2.1) where the $a_{n}$ and $b_{n}$ are polynomials in $n$, and secondly, in showing that $B_{n}$ and $G_{n}$ do indeed satisfy $\lim _{n \rightarrow \infty} G_{n} / B_{n}=0$.

Here we describe a very simple way of using Pincherle's theorem to write down infinite families of continued fractions such that each member of the family converges to the same limit. Moreover, $\lim _{n \rightarrow \infty} G_{n} / B_{n}=0$ is satisfied automatically and it is not necessary to have $a_{n}$ and $b_{n}$ polynomials in $n$. Initially the $a_{n}$ 's and $b_{n}$ 's are rational functions but $\mathrm{K}_{n=1}^{\infty} a_{n} / b_{n}$ can be converted to a polynomial continued fraction by a similarity transformation.

We first need some notation. If $f_{n}=g_{n} / h_{n}$ is a rational function in $n$, where $g_{n}$ and $h_{n}$ are polynomials in $n$, we define the degree of $f_{n}$ to be the degree of $g_{n}$ minus the degree of $h_{n}$ and we define the leading coefficient of $f_{n}$ to be the quotient of the leading coefficient of $g_{n}$ and the leading coefficient of $h_{n}$.

Proposition 1. Let $H_{n}$ and $b_{n}$ be rational functions in $n$ such that $H_{n}>0$ for $n \geq-1$ and $b_{n}>0$ for $n \geq 1$. We further assume that $b_{n}$ has degree greater than 0 or that, if it has degree 0 , then its leading coefficient is greater than 1 . Then the continued fraction

$$
{\underset{\mathrm{K}}{\mathrm{K}} \mathrm{\infty}}_{\frac{\left(H_{n}+b_{n} H_{n-1}\right) / H_{n-2}}{b_{n}}}
$$

converges and its limit is $H_{0} / H_{-1}$.

Proof. Let $G_{n}=(-1)^{n} H_{n}$ and $a_{n}=\left(H_{n}+b_{n} H_{n-1}\right) / H_{n-2}$. The sequences $\left\{G_{n}\right\}_{n=-1}^{\infty},\left\{a_{n}\right\}_{n=-1}^{\infty}$ and $\left\{b_{n}\right\}_{n=-1}^{\infty}$ are easily seen to satisfy equation (2.1). Furthermore, the recurrence relations at (1.3) and the conditions on the $b_{n}$ entail that $B_{n}$ grows at least as fast as either $D^{n}$ for some fixed $D>1$, or $(n !)^{\delta}$ for some fixed $\delta>0$. Since $G_{n}$ is of polynomial growth, it follows that $\lim _{n \rightarrow \infty} G_{n} / B_{n}=0$. Thus the continued fraction at (2.2) converges and its limit is $-G_{0} / G_{-1}=H_{0} / H_{-1}$.

As an illustration, we have the following example.

EXAMPLE 2. Let $H_{n}=n+2$ and let $b_{n}$ be any polynomial in $n$ such that $b_{n} \geq 2$ for $n \geq 1$. Then

$$
\begin{aligned}
& 2= \frac{3+2 b_{1}}{b_{1}}+\frac{4+3 b_{2}}{2 b_{2}}+\frac{2\left(5+4 b_{3}\right)}{3 b_{3}}+\cdots \\
&+\frac{(n-1)\left(n+2+(n+1) b_{n}\right)}{n b_{n}}+\cdots
\end{aligned}
$$

This follows from Proposition 1, after a similarity transformation to clear denominators.

REMARKS. 1) The restrictions that $H_{n}>0$ and $b_{n}>0$ are not so severe since, if we restrict to polynomial continued fractions $\mathrm{K}_{n=1}^{\infty} a_{n} / b_{n}$ in which 
the polynomials $a_{n}$ and $b_{n}$ have positive leading coefficients, then some tail of the continued fraction will have all $a_{n}, b_{n}>0$ so that, if one can find the limit of the tail, the continued fraction $\mathrm{K}_{n=1}^{\infty} a_{n} / b_{n}$ reduces to a finite continued fraction.

2) The limit of the continued fraction is independent of $b_{n}$ so that one has an infinite class of continued fractions converging to the same limit.

3) Although we restrict $H_{n}$ and $b_{n}$ to be rational functions of $n$, the result can easily be seen to be true for more general sequences of positive numbers, $\left\{H_{n}\right\}$ and $\left\{b_{n}\right\}$, provided the $b_{n}$ satisfy certain size or growth conditions.

4) Convergence to the stated limit may also hold for other polynomial sequences $\left\{b_{n}\right\}$ which do not satisfy the conditions of the proposition (for example, the continued fraction in Example 2 converges when $b_{n}$ is the constant polynomial 1).

We next write the rational functions $H_{n}$ and $b_{n}$ in terms of the polynomials defining them to obtain a result about polynomial continued fractions. This allows greater flexibility in deriving the limits of infinite families of polynomial continued fractions.

Corollary 1. Let $f_{n}, g_{n}, c_{n}$ and $d_{n}$ be polynomials in $n$ such that $f_{n}, g_{n} \neq 0$ for $n \geq-1, f_{n}$ and $g_{n}$ have the same sign for each $n \geq-1$, $c_{n}, d_{n} \neq 0$ for $n \geq 0$ and $c_{n}$ and $d_{n}$ have the same sign for each $n \geq 0$. Suppose further that the degree of $c_{n}$ is greater than the degree of $d_{n}$ or, if the degrees are equal, that the leading coefficient of $c_{n}$ is greater than the leading coefficient of $d_{n}$. Then the continued fraction

$$
\begin{aligned}
& \frac{g_{-1}\left(d_{1} f_{1} g_{0}+c_{1} f_{0} g_{1}\right)}{c_{1} f_{-1} g_{0} g_{1}}+\frac{d_{1} f_{-1} g_{0}^{2}\left(d_{2} f_{2} g_{1}+c_{2} f_{1} g_{2}\right)}{c_{2} f_{0} g_{2}} \\
& +\frac{d_{2} f_{0} g_{1}\left(d_{3} f_{3} g_{2}+c_{3} f_{2} g_{3}\right)}{c_{3} f_{1} g_{3}}+\frac{d_{3} f_{1} g_{2}\left(d_{4} f_{4} g_{3}+c_{4} f_{3} g_{4}\right)}{c_{4} f_{2} g_{4}}+\cdots \\
& +\frac{d_{n-1} f_{n-3} g_{n-2}\left(d_{n} f_{n} g_{n-1}+c_{n} f_{n-1} g_{n}\right)}{c_{n} f_{n-2} g_{n}}+\cdots
\end{aligned}
$$

converges and its limit is $f_{0} g_{-1} /\left(g_{0} f_{-1}\right)$.

Proof. In Proposition 1, let $H_{n}=f_{n} / g_{n}$ and $b_{n}=c_{n} / d_{n}$. The continued fraction at (2.2) is equivalent to the continued fraction at (2.3), after a similarity transformation. The conditions of Proposition 1 are satisfied and the result follows.

REMARK. The limit is independent of the polynomials $c_{n}$ and $d_{n}$, provided they satisfy the conditions of the corollary.

If we let $f_{n}=n^{2}+1$ and $d_{n}=g_{n}=1$ we get the following example.

EXAMPLE 3 . Let $c_{n}$ be any polynomials in $n$ such that $c_{n} \geq 2$ for $n \geq 0$. Then 


$$
\begin{aligned}
\frac{1}{2}= & \frac{c_{1}+2}{2 c_{1}}+\frac{2\left(2 c_{2}+5\right)}{c_{2}}+\frac{\left(5 c_{3}+10\right)}{2 c_{3}}+\frac{2\left(10 c_{4}+17\right)}{5 c_{4}}+\frac{5\left(17 c_{5}+26\right)}{10 c_{5}}+\cdots \\
& +\frac{\left(1+(-3+n)^{2}\right)\left(\left(1+(-1+n)^{2}\right) c_{n}+\left(1+n^{2}\right)\right)}{\left(1+(-2+n)^{2}\right) c_{n}}+\cdots
\end{aligned}
$$

If we let $f_{n}=d_{n}=1$ and $g_{n}=c_{n}$ and cancel a factor of $c_{-1} / c_{0}$ from the continued fraction and its limit, we get the following result.

EXAMPLE 4 . Let $c_{n}$ be any polynomial in $n$ such that $c_{n} \geq 2$ for $n \geq-1$. Then

$$
1=\frac{c_{0}+c_{1}^{2}}{c_{1}^{2}}+\frac{c_{0}\left(c_{1}+c_{2}^{2}\right)}{c_{2}^{2}}+\cdots+\frac{c_{-2+n}\left(c_{-1+n}+c_{n}^{2}\right)}{c_{n}^{2}}+\cdots
$$

We next study a generalization of Euler's transformation of a series into a continued fraction.

3. The Euler transformation of an infinite series. In 1775 , Daniel Bernoulli [3] proved the following result (see, for example, [6, pp. 11-12]).

Proposition 2. Let $\left\{K_{0}, K_{1}, \ldots\right\}$ be a sequence of complex numbers such that $K_{i} \neq K_{i-1}$ for $i=1,2, \ldots$ Then $\left\{K_{0}, K_{1}, \ldots\right\}$ is the sequence of approximants of the continued fraction

$$
\begin{aligned}
K_{0}+\frac{K_{1}-K_{0}}{1}+\frac{K_{1}-K_{2}}{K_{2}-K_{0}}+\frac{\left(K_{1}-K_{0}\right)\left(K_{2}-K_{3}\right)}{K_{3}-K_{1}}+\cdots \\
+\frac{\left(K_{n-2}-K_{n-3}\right)\left(K_{n-1}-K_{n}\right)}{K_{n}-K_{n-2}}+\cdots
\end{aligned}
$$

In particular, if $\left\{K_{n}\right\}$ is a convergent sequence, one gets a convergent continued fraction.

If one lets $K_{n}=\sum_{k=0}^{n} a_{k}$, one gets Euler's transformation of a series into a continued fraction [5]:

$$
\sum_{k=0}^{\infty} a_{k}=a_{0}+\frac{a_{1}}{1}+\frac{-a_{2}}{a_{1}+a_{2}}+\frac{-a_{1} a_{3}}{a_{2}+a_{3}}+\cdots+\frac{-a_{n-2} a_{n}}{a_{n-1}+a_{n}}+\cdots
$$

For example, applying Euler's transformation to the well known series for $\pi / 4$,

$$
\frac{\pi}{4}=\frac{1}{1}-\frac{1}{3}+\frac{1}{5}+\cdots+\frac{(-1)^{n-1}}{2 n-1}+\cdots,
$$

gives Lord Brouncker's continued fraction (1.1) (after inversion and some similarity transformations to clear fractions).

We now give a generalization of the Euler transformation.

Proposition 3. Let $\sum_{k=0}^{\infty} a_{k}$ be a convergent series and let $\left\{b_{n}\right\}$ be any sequence whose limit is zero such that $a_{n}+b_{n}-b_{n-1} \neq 0$ for $n \geq 1$. Then, 
for $N \geq 3$,

$$
\begin{aligned}
\sum_{i=0}^{N} a_{i}+b_{N}= & a_{0}+b_{0}+\frac{a_{1}+b_{1}-b_{0}}{1}+\frac{-a_{2}+b_{1}-b_{2}}{a_{2}+a_{1}+b_{2}-b_{0}} \\
& +{ }_{n=3}^{N} \frac{-\left(a_{n-2}+b_{n-2}-b_{n-3}\right)\left(a_{n}+b_{n}-b_{n-1}\right)}{a_{n}+a_{n-1}+b_{n}-b_{n-2}}
\end{aligned}
$$

and

$$
\begin{aligned}
\sum_{i=0}^{\infty} a_{i}= & a_{0}+b_{0}+\frac{a_{1}+b_{1}-b_{0}}{1}+\frac{-a_{2}+b_{1}-b_{2}}{a_{2}+a_{1}+b_{2}-b_{0}} \\
& +\stackrel{n}{K}_{n=3}^{\infty} \frac{-\left(a_{n-2}+b_{n-2}-b_{n-3}\right)\left(a_{n}+b_{n}-b_{n-1}\right)}{a_{n}+a_{n-1}+b_{n}-b_{n-2}} .
\end{aligned}
$$

Proof. This follows immediately from Proposition 2, upon letting $K_{n}=$ $\sum_{k=0}^{n} a_{k}+b_{n}$, noting that $\lim _{n \rightarrow \infty} K_{n}=\sum_{i=0}^{\infty} a_{i}$.

REMARK. It is easy to see that (3.4) holds for $N=0,1$, and 2 also, provided the right side is truncated at the zeroth, first and second approximant, respectively.

For our first example, we consider the series (3.3) for $\pi / 4$.

EXAMPLE 5. Let $f_{n}$ be any polynomial which is positive for $n \geq 1$ and, for ease of notation, define $g_{n}:=f_{n} f_{n-1}+(2 n-1)\left(f_{n}+f_{n-1}\right)$. Then

$$
\begin{aligned}
\frac{\pi}{4}= & \frac{-1}{f_{0}}+\frac{g_{1}}{f_{1} f_{0}}+\frac{f_{0}^{2} g_{2}}{2 f_{2} f_{0}+3\left(f_{2}-f_{0}\right)} \\
& +\stackrel{\mathrm{K}}{\mathrm{K}}_{n} \frac{(2 n-3)^{2} g_{n-2} g_{n}}{2 f_{n} f_{n-2}+(2 n-1)(2 n-3)\left(f_{n}-f_{n-2}\right)} .
\end{aligned}
$$

This follows from setting $b_{n}=(-1)^{n-1} / f_{n}$ in Proposition 3 and simplifying the continued fraction. If we let $f_{n}=A(2 n-1)$, where $A$ is a positive integer, we get, after some further simplification,

$$
\begin{aligned}
\frac{\pi}{4}= & \frac{1}{A}+\frac{1}{1}-\frac{4+A}{4-2 A} \\
& +{ }_{n=3}^{\infty} \frac{(2 n-3)(2 n-5)[A(2 n-7)+4(n-3)][A(2 n-3)+4(n-1)]}{2 A(2 n-5)+4(2 n-3)} .
\end{aligned}
$$

As a second example, we consider the series representation for $\zeta(k)$, $k$ an integer greater than 1 :

$$
\zeta(k)=\sum_{n=1}^{\infty} \frac{1}{n^{k}} .
$$

If the Euler transformation is applied to this series (letting $a_{0}=0$ and 
$a_{i}=1 / i^{k}$ for $i \geq 1$ ), one easily gets

$$
\zeta(k)=\frac{1}{1}-\frac{1}{2^{k}+1}-\frac{2^{2 k}}{3^{k}+2^{k}}-\frac{3^{2 k}}{4^{k}+3^{k}}-\cdots-\frac{(n-1)^{2 k}}{n^{k}+(n-1)^{k}}-\cdots
$$

If we set $b_{n}=1 / d_{n}$ in Proposition 3 , where $d_{n}$ is a polynomial of degree at least 1 such that $g_{n}:=d_{n} d_{n-1}+n^{k}\left(d_{n-1}-d_{n}\right) \neq 0$ for $n \geq 1$, then

$$
\begin{aligned}
\zeta(k)= & \frac{1}{d_{0}}+\frac{g_{1}}{d_{0} d_{1}}-\frac{d_{0}^{2} g_{2}}{d_{2} d_{0}\left(1+2^{k}\right)+2^{k}\left(d_{0}-d_{2}\right)} \\
& +{\underset{n=3}{\infty} \frac{-(n-1)^{2 k} g_{n-2} g_{n}}{d_{n} d_{n-2}\left((n-1)^{k}+n^{k}\right)+(n-1)^{k} n^{k}\left(d_{n-2}-d_{n}\right)}} .
\end{aligned}
$$

We can specialize further to get the following continued fraction.

EXAMPle 6 . If $A$ is a positive integer, then

$$
\begin{aligned}
\zeta(k)= & \frac{1}{A}+\frac{2 A-1}{2 A}-\frac{2 A\left(3 A-2^{k-1}\right)}{3 A\left(1+2^{k}\right)-2^{k+1}} \\
& +{ }_{n=3}^{\infty} \frac{-n(n-1)^{2 k-1}\left[A(n-1)-(n-2)^{k-1}\right]\left[A(n+1)-n^{k-1}\right]}{A(n+1)\left[(n-1)^{k}+n^{k}\right]-2 n^{k}(n-1)^{k-1}} .
\end{aligned}
$$

This follows from defining $d_{n}:=A(n+1)$ and simplifying the continued fraction.

For our third example, we consider the binomial series. Let $|x|<1$ and $\alpha \in \mathbb{R}$. Then

$$
\begin{aligned}
(1+x)^{\alpha} & =1+\alpha x+\frac{\alpha(\alpha-1)}{2 !} x^{2}+\frac{\alpha(\alpha-1)(\alpha-2)}{3 !} x^{3}+\cdots \\
& =\sum_{n=0}^{\infty} \frac{(\alpha)_{n}}{n !} x^{n}
\end{aligned}
$$

where $(\alpha)_{n}=\alpha(\alpha-1) \cdots(\alpha-n+1)$ denotes the falling factorial. If we let $b_{n}=r_{n} x^{n}(\alpha)_{n} / n$ !, where $r_{n}$ is any rational function such that

$$
g_{n}:=(\alpha-n+1) x\left(1+r_{n}\right)-n r_{n-1} \neq 0, \quad n \geq 1,
$$

then Proposition 3 gives

$$
\begin{aligned}
(1+x)^{\alpha} & =1+r_{0}+\frac{g_{1}}{1}-\frac{\alpha x g_{2}}{\alpha x\left[(\alpha-1) x\left(1+r_{2}\right)+2\right]-2 r_{0}} \\
& +{ }_{n=3}^{\infty} \frac{-(n-1) x(\alpha-n+2) g_{n-2} g_{n}}{(\alpha-n+2) x\left[(\alpha-n+1) x\left(1+r_{n}\right)+n\right]-n(n-1) r_{n-2}} .
\end{aligned}
$$

If we specialize by letting $\alpha=1 / 5, x=5 / 7$ and $r_{n}=A n-1(A \neq-7)$, and then simplifying the continued fraction, we get: 
EXAMPLE 7 . If $A$ is any integer different from -7 , then

$$
\begin{aligned}
\sqrt[5]{\frac{12}{7}=} & \frac{A+7}{7}+\frac{7(11 A-7)}{-4 A+56} \\
& +{ }_{n=3}^{\infty} \frac{7(5 n-11)(n-2)[(12 n-37) A-7][(12 n-13) A-7]}{-2 A\left(12 n^{2}-31 n+16\right)+14(2+n)} .
\end{aligned}
$$

REMARK. It follows from equation (3.7) that every real number of the form $(p / q)^{r / s}$, where $p, q, r$ and $s$ are integers, can be expanded in infinitely many ways as a polynomial continued fraction.

We next consider a generalization of the formula transforming an infinite product to a continued fraction.

\section{The transformation of infinite products to continued frac-} tions. In equation (3.1), if $K_{i} \neq 0$ for $i \geq 1$, then the continued fraction on the left side can be re-written as

$$
\begin{aligned}
K_{0}+\frac{K_{1}-K_{0}}{1}-\frac{K_{1} / K_{0}\left(K_{2} / K_{1}-1\right)}{K_{2} / K_{0}-1} & -\cdots \\
& -\frac{K_{2} / K_{1}\left(K_{1} / K_{0}-1\right)\left(K_{3} / K_{2}-1\right)}{K_{3} / K_{1}-1}-\ldots \\
& -\frac{K_{n-1} / K_{n-2}\left(K_{n-2} / K_{n-3}-1\right)\left(K_{n} / K_{n-1}-1\right)}{K_{n} / K_{n-2}-1}-\cdots
\end{aligned}
$$

In particular, if $K_{0}=1$ and $K_{n}=\prod_{i=1}^{n} a_{i}$ for $n \geq 1$, where $\prod_{i=1}^{\infty} a_{i}$ is a convergent infinite product with no $a_{i}=0$ or 1 , one has

$$
\prod_{i=1}^{\infty} a_{i}=1+\frac{a_{1}-1}{1}-\frac{a_{1}\left(a_{2}-1\right)}{a_{2} a_{1}-1}+{\underset{n=3}{K}}_{n}^{\infty} \frac{-a_{n-1}\left(a_{n-2}-1\right)\left(a_{n}-1\right)}{a_{n} a_{n-1}-1} .
$$

This transformation is not so well known as Euler's transformation of an infinite series to a continued fraction. As with the Euler transformation, it is easy to generalize the transformation at (4.2).

Proposition 4. Let $\prod_{i=1}^{\infty} a_{i}$ be a convergent infinite product with $a_{i} \neq 0$ for $i \geq 1$ and let $\left\{b_{n}\right\}_{n=0}^{\infty}$ be any sequence whose limit is 1 such that $a_{i} b_{i}-b_{i-1} \neq 0$ for $i \geq 1$. Then

$$
\begin{aligned}
\prod_{i=1}^{\infty} a_{i}= & b_{0}+\frac{a_{1} b_{1}-b_{0}}{1}-\frac{a_{1}\left(a_{2} b_{2}-b_{1}\right)}{a_{2} a_{1} b_{2}-b_{0}} \\
& +\stackrel{\mathrm{K}}{\mathrm{K}}^{\mathrm{-}} \frac{-a_{n-1}\left(a_{n-2} b_{n-2}-b_{n-3}\right)\left(a_{n} b_{n}-b_{n-1}\right)}{a_{n} a_{n-1} b_{n}-b_{n-2}} .
\end{aligned}
$$

Proof. This follows from Proposition 2 upon setting $K_{0}=b_{0}$ and, for $n \geq 1$, setting $K_{n}=b_{n} \prod_{i=1}^{n} a_{i}$ and then simplifying the continued fraction. 
As an example, we consider the following infinite product identity:

$$
\frac{\sin \pi x}{\pi x}=\prod_{n=1}^{\infty}\left(1-\frac{x^{2}}{n^{2}}\right) .
$$

Set $x=1 / m$, where $m$ is a positive integer, and $b_{n}=1+A /(n+1)$, where $A$ is an integer. For ease of notation, let

$$
\begin{aligned}
& g_{n}=\left(m^{2} n+1\right) A+(n+1), \\
& h_{n}=\left(m^{2} n^{2}-1\right)\left(m^{2}(n-1)^{2}-1\right)(A+n+1)-m^{4} n^{2}\left(n^{2}-1\right)(A+n-1) .
\end{aligned}
$$

Proposition 4.3 then shows, after simplifying the continued fraction, that

$$
\begin{aligned}
\frac{m \sin \pi / m}{\pi} & =1+A+\frac{\left(m^{2}-1\right)(A+2)-2 m^{2}(A+1)}{2 m^{2}} \\
& +\frac{2 m^{2}\left(m^{2}-1\right) g_{2}}{h_{2}}+\underset{n=3}{K} \frac{-n(n-1) m^{2}\left(m^{2}(n-1)^{2}-1\right) g_{n-2} g_{n}}{h_{n}} .
\end{aligned}
$$

If we specialize further and let $m=3$, we have

Example 8. For each integer $A$,

$$
\begin{aligned}
& \frac{3 \sqrt{3}}{2 \pi}=1+A+\frac{-10 A-2}{18}+\frac{2736 A+432}{-692 A-132} \\
& +{ }_{n=3}^{\infty} \frac{9 n(1-n)(3 n-2)(3 n-4)[(1+9 A) n+A+1][(1+9 A) n-17 A-1]}{-8(1+A)+2(5+9 A) n+144 A n^{2}-18(1+9 A) n^{3}} .
\end{aligned}
$$

\section{Extensions, contractions and the Bauer-Muir transforma-} tion. We start with the concepts of extensions and contractions of continued fractions. Before coming to details, we borrow some notation from [7] (page 83). A continued fraction $d_{0}+\mathrm{K}_{n=1}^{\infty} c_{n} / d_{n}$ is said to be a contraction of the continued fraction $b_{0}+\mathrm{K}_{n=1}^{\infty} a_{n} / b_{n}$ if its classical approximants $\left\{g_{n}\right\}$ form a subsequence of the classical approximants $\left\{f_{n}\right\}$ of $b_{0}+\mathrm{K}_{n=1}^{\infty} a_{n} / b_{n}$. In this case $b_{0}+\mathrm{K}_{n=1}^{\infty} a_{n} / b_{n}$ is called an extension of $d_{0}+\mathrm{K}_{n=1}^{\infty} c_{n} / d_{n}$.

We call $d_{0}+\mathrm{K}_{n=1}^{\infty} c_{n} / d_{n}$ a canonical contraction of $b_{0}+\mathrm{K}_{n=1}^{\infty} a_{n} / b_{n}$ if

$$
C_{k}=A_{n_{k}}, \quad D_{k}=B_{n_{k}} \quad \text { for } k=0,1, \ldots,
$$

where $C_{n}, D_{n}, A_{n}$ and $B_{n}$ are canonical numerators and denominators of $d_{0}+\mathrm{K}_{n=1}^{\infty} c_{n} / d_{n}$ and $b_{0}+\mathrm{K}_{n=1}^{\infty} a_{n} / b_{n}$ respectively.

From [7] (pages 83 and 85) we also have the following theorems.

THEOREM 2. The canonical contraction of $b_{0}+\mathrm{K}_{n=1}^{\infty} a_{n} / b_{n}$ with

$$
C_{k}=A_{2 k}, \quad D_{k}=B_{2 k} \quad \text { for } k=0,1, \ldots
$$

exists if and only if $b_{2 k} \neq 0$ for $K=1,2, \ldots$, and in this case is given by

$$
b_{0}+\frac{b_{2} a_{1}}{b_{2} b_{1}+a_{2}}-\frac{a_{2} a_{3} b_{4} / b_{2}}{a_{4}+b_{3} b_{4}+a_{3} b_{4} / b_{2}}-\frac{a_{4} a_{5} b_{6} / b_{4}}{a_{6}+b_{5} b_{6}+a_{5} b_{6} / b_{4}}+\cdots
$$

The continued fraction (5.1) is called the even part of $b_{0}+\mathrm{K}_{n=1}^{\infty} a_{n} / b_{n}$. 
THEOREM 3. The canonical contraction of $b_{0}+\mathrm{K}_{n=1}^{\infty} a_{n} / b_{n}$ with $C_{0}=$ $A_{1} / B_{1}$,

$$
C_{k}=A_{2 k+1}, \quad D_{k}=B_{2 k+1} \quad \text { for } k=1,2, \ldots
$$

exists if and only if $b_{2 k+1} \neq 0$ for $K=0,1,2, \ldots$, and in this case is given by

$$
\begin{aligned}
\frac{b_{0} b_{1}+a_{1}}{b_{1}}- & \frac{a_{1} a_{2} b_{3} / b_{1}}{b_{1}\left(a_{3}+b_{2} b_{3}\right)+a_{2} b_{3}}-\frac{a_{3} a_{4} b_{5} b_{1} / b_{3}}{a_{5}+b_{4} b_{5}+a_{4} b_{5} / b_{3}} \\
& -\frac{a_{5} a_{6} b_{7} / b_{5}}{a_{7}+b_{6} b_{7}+a_{6} b_{7} / b_{5}}-\frac{a_{7} a_{8} b_{9} / b_{7}}{a_{9}+b_{8} b_{9}+a_{8} b_{9} / b_{7}}+\cdots
\end{aligned}
$$

The continued fraction (5.2) is called the odd part of $b_{0}+\mathrm{K}_{n=1}^{\infty} a_{n} / b_{n}$.

One might expect that if a continued fraction is constructed whose even part is the convergent continued fraction $\mathrm{K}_{n=1}^{\infty} a_{n} / b_{n}$, one might have some flexibility in the choice of the elements of the extended continued fraction. This is indeed the case and if, in addition, the extended continued fraction can be constructed so that it too converges, an infinite family of continued fractions with the same limit as $\mathrm{K}_{n=1}^{\infty} a_{n} / b_{n}$ can be found.

However, before discussing this we first consider another way of transforming a continued fraction so as to produce an infinite family of continued fractions with the same limit.

Definition ([7, p. 76]). The Bauer-Muir transform of a continued fraction $b_{0}+K\left(a_{n} / b_{n}\right)$ with respect to a sequence $\left\{w_{n}\right\}$ from $\mathbb{C}$ is the continued fraction $d_{0}+K\left(c_{n} / d_{n}\right)$ whose canonical numerators $C_{n}$ and denominators $D_{n}$ are given by

$$
C_{-1}=1, \quad C_{n}=A_{n}+w_{n} A_{n-1}, \quad D_{-1}=0, \quad D_{n}=B_{n}+w_{n} B_{n-1}
$$

for $n=0,1, \ldots$, where $\left\{A_{n}\right\}$ and $\left\{B_{n}\right\}$ are the canonical numerators and denominators of $b_{0}+K\left(a_{n} / b_{n}\right)$.

This transformation dates back to the 1870's and the work of Bauer [1] and Muir [8]. From [7, p. 76], there is the following theorem:

THEOREM 4. The Bauer-Muir transform of $b_{0}+K\left(a_{n} / b_{n}\right)$ with respect to $\left\{w_{n}\right\}$ from $\mathbb{C}$ exists if and only if

$$
a_{n}-w_{n-1}\left(b_{n}+w_{n}\right) \neq 0 \quad \text { for } n=1,2, \ldots
$$

If it exists, then it is given by

$$
b_{0}+w_{0}+\frac{a_{1}-w_{0}\left(b_{1}+w_{1}\right)}{b_{1}+w_{1}}+\frac{c_{2}}{d_{2}}+\frac{c_{3}}{d_{3}}+\cdots
$$

where 


$$
\begin{aligned}
& c_{n}=a_{n-1} \frac{a_{n}-w_{n-1}\left(b_{n}+w_{n}\right)}{a_{n-1}-w_{n-2}\left(b_{n-1}+w_{n-1}\right)}, \\
& d_{n}=b_{n}+w_{n}-w_{n-2} \frac{a_{n}-w_{n-1}\left(b_{n}+w_{n}\right)}{a_{n-1}-w_{n-2}\left(b_{n-1}+w_{n-1}\right)} .
\end{aligned}
$$

We have changed the notation found in [7] slightly. One might expect that, by choosing the sequence $\left\{w_{n}\right\}$ appropriately, one could construct an infinite family of continued fractions with the same limit as the convergent continued fraction $K\left(a_{n} / b_{n}\right)$.

Curiously, these two methods (extensions and contractions and the Bauer-Muir transform) of producing infinite families of continued fractions in which each continued fraction has the same limit as the convergent continued fraction $K\left(a_{n} / b_{n}\right)$ are related. We believe the following observation to be new.

Theorem 5. Let $\left\{w_{n}\right\}_{n=0}^{\infty}$ be a sequence from $\mathbb{C}$ such that $w_{0}=0$ and $w_{n} \neq 0$ for $n \geq 1$. Suppose further that $\left\{a_{n}\right\}_{n=1}^{\infty}$ and $\left\{b_{n}\right\}_{n=1}^{\infty}$ are sequences from $\mathbb{C}$ such that $a_{n}-w_{n-1}\left(b_{n}+w_{n}\right) \neq 0$ for $n=1,2, \ldots$ Then the even part of the continued fraction

$$
\begin{aligned}
\frac{a_{1}}{b_{1}+w_{1}}+ & \frac{-w_{1}}{1} \\
& +\frac{a_{2} / w_{1}}{b_{2}+w_{2}-a_{2} / w_{1}}+\frac{-w_{2}}{1}+\frac{a_{3} / w_{2}}{b_{3}+w_{3}-a_{3} / w_{2}}+\cdots \\
& +\frac{-w_{n-1}}{1}+\frac{a_{n} / w_{n-1}}{b_{n}+w_{n}-a_{n} / w_{n-1}}+\frac{-w_{n}}{1}+\cdots
\end{aligned}
$$

is $\mathrm{K}_{n=1}^{\infty} a_{n} / b_{n}$ and its odd part is equal to the Bauer-Muir transform of $\mathrm{K}_{n=1}^{\infty} a_{n} / b_{n}$ with respect to the sequence $\left\{w_{n}\right\}_{n=0}^{\infty}$.

REMARK. The theorem says that the odd part of the continued fraction at (5.7) "is equal to" the Bauer-Muir transform of $\mathrm{K}_{n=1}^{\infty} a_{n} / b_{n}$ with respect to the sequence $\left\{w_{n}\right\}_{n=0}^{\infty}$ rather than saying that it "is" this Bauer-Muir transform, since some transformations need to be applied to the odd part to make it equal the stated Bauer-Muir transform of $\mathrm{K}_{n=1}^{\infty} a_{n} / b_{n}$.

Proof of Theorem 5. That the even part of (5.7) is $\mathrm{K}_{n=1}^{\infty} a_{n} / b_{n}$ follows immediately from Theorem 2. From Theorem 3, the odd part of (5.7) is

$$
\frac{a_{1}}{b_{1}+w_{1}}-\frac{a_{1}\left(-w_{1}\right) \frac{b_{2}+w_{2}-a_{2} / w_{1}}{b_{1}+w_{1}}}{\left(b_{1}+w_{1}\right)\left[\frac{a_{2}}{w_{1}}+\left(b_{2}+w_{2}-\frac{a_{2}}{w_{1}}\right)\right]-w_{1}\left(b_{2}+w_{2}-a_{2} / w_{1}\right)}
$$




$$
\begin{gathered}
-\frac{a_{2} / w_{1}\left(-w_{2}\right) \frac{b_{3}+w_{3}-a_{3} / w_{2}}{b_{2}+w_{2}-a_{2} / w_{1}}\left(b_{1}+w_{1}\right)}{a_{3} / w_{2}+\left(b_{3}+w_{3}-a_{3} / w_{2}\right)-w_{2} \frac{b_{3}+w_{3}-a_{3} / w_{2}}{b_{2}+w_{2}-a_{2} / w_{1}}} \\
-\frac{a_{3} / w_{2}\left(-w_{3}\right) \frac{b_{4}+w_{4}-a_{4} / w_{3}}{b_{3}+w_{3}-a_{3} / w_{2}}}{a_{4} / w_{3}+\left(b_{4}+w_{4}-a_{4} / w_{3}\right)-w_{3} \frac{b_{4}+w_{4}-a_{4} / w_{3}}{b_{3}+w_{3}-a_{3} / w_{2}}-\cdots} \frac{a_{n}+w_{n}-a_{n} / w_{n-1}}{\left.a_{n-1} / w_{n-2}\left(-w_{n-1}\right) \frac{b_{n-1}+w_{n-1}-a_{n-1} / w_{n-2}}{b_{n}}\right)-w_{n-1} \frac{b_{n}+w_{n}-a_{n} / w_{n-1}}{b_{n-1}+w_{n-1}-a_{n-1} / w_{n-2}}}
\end{gathered}
$$

Since $b_{0}=w_{0}=0$, all that is necessary to complete the proof is to show that, for $\alpha$ arbitrary,

$$
\frac{a_{1}}{b_{1}+w_{1}}+\frac{a_{2}-w_{1}\left(b_{2}+w_{2}\right)}{b_{2}+w_{2}+\alpha}=\frac{a_{1}}{b_{1}+w_{1}}-\frac{a_{1} \frac{a_{2}-w_{1}\left(b_{2}+w_{2}\right)}{\left(b_{1}+w_{1}\right)^{2}}}{\frac{a_{2}+b_{1}\left(b_{2}+w_{2}\right)}{b_{1}+w_{1}}+\alpha} .
$$

This is immediate from the identity

$$
\frac{E}{F}+\frac{G}{H+\alpha}=\frac{E}{F}-\frac{E G / F^{2}}{(F H+G) / F+\alpha} .
$$

We next consider the question of when a continued fraction and its Bauer-Muir transform converge to the same limit. One approach is to apply general convergence theorems to the continued fraction at (5.7). If this 
continued fraction converges, then its odd and even parts converge to the same limit. Alternatively, we have the following proposition.

Proposition 5. Let $\left\{w_{n}\right\}_{n=0}^{\infty}$ be any rational function sequence taking only positive values for $n \geq 1$ and suppose $w_{0}=0$. Suppose further that the polynomial sequences $\left\{a_{n}\right\}_{n=1}^{\infty}$ and $\left\{b_{n}\right\}_{n=1}^{\infty}$ take only positive values for $n \geq 1$, that $a_{n}-w_{n-1}\left(b_{n}+w_{n}\right) \neq 0$ for $n=1,2, \ldots$ and that the continued fraction $\mathrm{K}_{n=1}^{\infty} a_{n} / b_{n}$ converges. Then the Bauer-Muir transform of $\mathrm{K}_{n=1}^{\infty} a_{n} / b_{n}$ with respect to $\left\{w_{n}\right\}_{n=0}^{\infty}$ converges to the same limit.

Proof. Suppose $\mathrm{K}_{n=1}^{\infty} a_{n} / b_{n}=L$. Then

$$
\lim _{n \rightarrow \infty} L-\frac{A_{n}}{B_{n}}=\lim _{n \rightarrow \infty} \frac{A_{n+1}}{B_{n+1}}-\frac{A_{n}}{B_{n}}=0
$$

and

$$
\begin{aligned}
\left|L-\frac{A_{n+1}+w_{n} A_{n}}{B_{n+1}+w_{n} B_{n}}\right| & \leq\left|L-\frac{A_{n}}{B_{n}}\right|+\left|\frac{A_{n+1}+w_{n} A_{n}}{B_{n+1}+w_{n} B_{n}}-\frac{A_{n}}{B_{n}}\right| \\
& =\left|L-\frac{A_{n}}{B_{n}}\right|+\left|\frac{A_{n+1}}{B_{n+1}}-\frac{A_{n}}{B_{n}}\right| \frac{1}{1+w_{n} B_{n} / B_{n+1}} \\
& \leq\left|L-\frac{A_{n}}{B_{n}}\right|+\left|\frac{A_{n+1}}{B_{n+1}}-\frac{A_{n}}{B_{n}}\right| .
\end{aligned}
$$

The last inequality follows since $w_{n} B_{n} / B_{n+1} \geq 0$. Finally, let $n \rightarrow \infty$ to get the result.

This can be restated as follows: If $a_{n}$ and $b_{n}$ are polynomials taking only positive values for $n \geq 1$ and $w_{n}$ is a rational function taking only positive values for $n \geq 1$ and $w_{0}$ is defined to be 0 , then

$$
\begin{aligned}
\stackrel{\infty}{\mathrm{K}} \frac{a_{n}}{b_{n}}= & \frac{a_{1}}{b_{1}+w_{1}}+\frac{a_{1}\left(a_{2}-w_{1}\left(b_{2}+w_{2}\right)\right)}{a_{1}\left(b_{2}+w_{2}\right)} \\
& +\underset{n=3}{\mathrm{~K}} \frac{a_{n-1}\left[a_{n}-w_{n-1}\left(b_{n}+w_{n}\right)\right]\left[a_{n-2}-w_{n-3}\left(b_{n-2}+w_{n-2}\right)\right]}{a_{n-1}\left(b_{n}+w_{n}\right)-w_{n-2}\left(a_{n}+b_{n-1}\left(b_{n}+w_{n}\right)\right)} .
\end{aligned}
$$

As an example, we consider the well known continued fraction expansion for $e$ :

$$
e=2+\frac{2}{2}+\frac{3}{3}+\frac{4}{4}+\cdots+\frac{n}{n}+\cdots
$$

If we let $A$ be a non-negative integer and define $w_{0}:=0$ and $w_{n}:=A(n+1)$ for $n \geq 1$, we get the example below.

EXAMPLE 9. If $A$ is a non-negative integer, then

$$
\begin{aligned}
e=2 & +\frac{1}{1+A}+\frac{1-2 A(1+A)}{2(1+A)}+\frac{2(1-3 A(1+A))}{3-5 A-6 A^{2}} \\
& +{\underset{K}{\infty} \frac{\infty}{(n-1)[1-n A(1+A)][1-(n-2) A(1+A)]}}_{n-(n(n-1)-1) A-n(n-1) A^{2}}
\end{aligned}
$$


6. Conclusion. Let $\mathbb{P}$ denote the set of all polynomial continued fractions $\left({ }^{2}\right)$. It is not difficult to construct an injective map from $\mathbb{P}$ to $\mathbb{N}$, so that $\mathbb{P}$ is a countable set and thus, almost all real numbers do not have a polynomial continued fraction expansion. Let $\mathbb{P}^{\prime}$ denote the set of real numbers which are the limits of convergent continued fractions in $\mathbb{P}$. Trivially, $\mathbb{Q} \in \mathbb{P}^{\prime}$ and, as is shown by examples from the literature, many algebraic and transcendental numbers also have a polynomial continued fraction expansion. We conclude with a number of questions about polynomial continued fractions which we consider interesting.

1 ) Is there an equivalent classification of the set $\mathbb{P}^{\prime}$, perhaps in terms of the partial quotients in the regular continued fraction expansion of its elements or in terms of irrationality measure?

2) Does every real algebraic number belong to $\mathbb{P}^{\prime}$ ? If not, exhibit a counter-example. Note that the answer to this second question is yes if every real algebraic number $\alpha$ can be expanded as a hypergeometric series $\alpha=\sum_{i=0}^{\infty} b_{i}$, where each $b_{i} \in \mathbb{Q}$ and $b_{n+1} / b_{n}=r(n)$, where $r(x) \in \mathbb{Q}(x)$. We are not aware if this question has been answered in the literature.

3) Since almost all real numbers do not belong to $\mathbb{P}^{\prime}$, exhibit an example of such a number, or perhaps an infinite family of such numbers.

4) With a little more effort, Proposition 5 can be extended to show that, given any convergent polynomial continued fraction, there is an infinite family of convergent polynomial continued fractions with the same limit. Let $\mathbb{P}_{n, d}$ denote the set of all polynomial continued fractions of degree at most $n$ in the numerator and degree at most $d$ in the denominator. Does there exist a pair of non-negative integers $n$ and $d$ such that every element of $\mathbb{P}$ that converges has the same limit as some element of $\mathbb{P}_{n, d}$ ? Is this true for $n=2$ and $d=1\left({ }^{3}\right)$ ?

5) Trivially, if $\alpha \neq 0$ and $\alpha \in \mathbb{P}^{\prime}$, then $1 / \alpha \in \mathbb{P}^{\prime}$. Does $\mathbb{P}^{\prime}$ have any further algebraic structure? Is it true that if $\alpha, \beta \in \mathbb{P}^{\prime}$, then $\alpha+\beta \in \mathbb{P}^{\prime}$ ? Is it true that if $\alpha, \beta \in \mathbb{P}^{\prime}$, then $\alpha \times \beta \in \mathbb{P}^{\prime}$ ? Even negative answers to these questions would be of interest but it would probably not be easy to find counter-examples.

\section{References}

[1] F. Bauer, Von einem Kettenbruche Euler's und einem Theorem von Wallis, Abh. Königl. Bayer. Akad. Wiss. München II Kl. 11 (1872), 99-116.

[2] B. C. Berndt, Ramanujan's Notebook's, Part II, Springer, New York, 1989.

$\left({ }^{2}\right)$ Of course not every element of $\mathbb{P}$ converges to a real number.

$\left({ }^{3}\right)$ This would be of interest since every element of $\mathbb{P}_{2,1}$ can be evaluated in terms of hypergeometric functions. 
[3] D. Bernoulli, Disquisitiones ulteriores de indole fractionum continuarum, Novi Comment. Acad. Sci. Imper. Petropol. 20 (1775), 9-11.

[4] D. Bowman and J. Mc Laughlin, Polynomial continued fractions, Acta Arith. 103 (2002), 329-342.

[5] L. Euler, De transformatione serierum in fractiones continuas, ubi simul haec theoria non mediocriter amplificatur, in: Omnia Opera, Ser. I, Vol. 15, Teubner, Lipsiae, 1927, 661-700.

[6] A. N. Khovanskiı̌, The Application of Continued Fractions and their Generalizations to Problems in Approximation Theory, translated by Peter Wynn, Noordhoff, Groningen, 1963.

[7] L. Lorentzen and H. Waadeland, Continued Fractions with Applications, NorthHolland, Amsterdam, 1992.

[8] T. Muir, A Theorem in continuants, Phil. Mag. (5) 3 (1877), 137-138.

[9] O. Perron, Die Lehre von den Kettenbrüchen, Teubner, Leipzig, 1913.

[10] S. Pincherle, Delle funzioni ipergeometriche e di varie questioni ad esse attinenti, Giorn. Mat. Battaglini 32 (1894), 209-291.

[11] J. Wallis, Arithmetica infinitorum, sive nova methodus inquirendi in curvilineorum quadraturam, aliaque difficiliora matheseos problemata, Oxford, 1655.

Department of Mathematics

Trinity College

300 Summit Street

Hartford, CT 06106-3100, U.S.A.

E-mail: james.mclaughlin@trincoll.edu nancy.wyshinski@trincoll.edu

Received on 1.3.2004

and in revised form on 16.8.2004 